\title{
Optical Absorption and Emission Properties of End- capped Oligothienoacenes: A Joint Theoretical and Experimental Study
}

\section{Citation}

Sánchez-Carrera, Roel S., M. Carmen Ruiz Delgado, Cristina Capel Ferrón, Reyes Malavé Osuna, Victor Hernández, Juan T. López Navarrete, and Alán Aspuru-Guzik. 2010. Optical absorption and emission properties of end-capped oligothienoacenes: a joint theoretical and experimental study. Organic Electronics 11(10): 1701-1712.

\section{Published Version}

http://dx.doi.org/10.1016/j.orgel.2010.07.001

\section{Permanent link}

http://nrs.harvard.edu/urn-3:HUL.InstRepos:9637920

\section{Terms of Use}

This article was downloaded from Harvard University's DASH repository, and is made available under the terms and conditions applicable to Open Access Policy Articles, as set forth at http:// nrs.harvard.edu/urn-3:HUL.InstRepos:dash.current.terms-of-use\#OAP

\section{Share Your Story}

The Harvard community has made this article openly available.

Please share how this access benefits you. Submit a story.

Accessibility 


\title{
Optical Absorption and Emission Properties of End-capped
}

\section{Oligothienoacenes: A Joint Theoretical and Experimental Study}

\author{
Roel S. Sánchez-Carrera, ${ }^{*[a]}$ M. Carmen Ruiz Delgado, ${ }^{*[b, c]}$ Cristina Capel Ferrón, ${ }^{[c]}$ \\ Reyes Malavé Osuna, ${ }^{[c]}$ Víctor Hernández, ${ }^{*[c]}$ Juan T. López Navarrete, ${ }^{*[c]}$ \\ and Alán Aspuru-Guzik.*[a]
}

[a] Dr. R. S. Sánchez-Carrera, Prof. A. Aspuru-Guzik Department of Chemistry and Chemical Biology Harvard University Cambridge, Massachusetts 02138 (USA)

E-mail: $\quad$ roel@gatech.edu aspuru@ chemistry.harvard.edu

[b] Dr. M. C. Ruiz Delgado, School of Chemistry and Biochemistry and Center for Organic Photonics and Electronics Georgia Institute of Technology Atlanta, GA 30332-0400 (USA)

[c] C. Capel Ferrón, Dr. M. C. Ruiz Delgado, Dr. R. Malavé Osuna, Dr. V. Hernández, Prof. J. T. López Navarrete

Departamento de Química Física

Universidad de Málaga

Málaga 29071 (Spain)

E-mail: hernandez@uma.es

teodomiro@uma.es

carmenrd@uma.es

Keywords: oligothienoacenes, optical properties, reorganization energy, vibronic coupling, time-dependent density functional calculations 


\section{Abstract}

The electron-vibration coupling in a family of silyl end-capped oligothienoacenes (TIPS-TnTIPS, $n=4-8$ ) is studied on the basis of a joint experimental and theoretical study using UV-Vis absorption and emission spectroscopies and density functional theory (DFT) calculations. Wellresolved vibronic progressions are found in the low-temperature absorption and emission profiles of these silyl-functionalized organic molecules. As the size of the oligomer lengthens a bathochromic shift is observed in the near-UV-Visible range, indicative of the extension of the effective $\pi$-conjugation. The absorption and emission bands are practically mirror-symmetric. The combination of two normal modes with frequencies of $\sim 1500 \mathrm{~cm}^{-1}$ and $\sim 500 \mathrm{~cm}^{-1}$ determines the main vibronic progression in absorption and emission for all the series, although for larger oligomers $(n=6,7$, and 8$)$ the presence of low-frequency normal modes $\left(\sim 100 \mathrm{~cm}^{-1}\right)$ is also evident. The spacing of the vibrational features is slightly larger in absorption than in emission; this agrees with the predicted shifting of the $\mathrm{C}-\mathrm{C}$ stretching modes of the inner-most ring toward the high-frequency region as a result of the reversal of the single-double $\mathrm{C}-\mathrm{C}$ pattern in the electronic excited state. Our calculations indicate that the contributions of the TIPS groups to the total relaxation energy of the $\mathrm{S}_{0} \rightarrow \mathrm{S}_{1}$ and $\mathrm{S}_{1} \rightarrow \mathrm{S}_{0}$ transitions are almost negligible. This result suggest that the vibronic structure and to a large extent the spectral profiles of the silyl end-capped oligothienoacenes are mainly determined by their respective oligothienyl core. 


\section{Introduction}

Currently, $\pi$-conjugated oligomers, such as oligoacenes and oligothiophenes are amongst the most widely studied molecular materials involved in the development of the next generation of optoelectronic devices such organic solar cells and organic field-effect transistors.[1-4] Among the many different molecular materials for organic electronic applications, oligothienoacenes (linearly fused thiophenes) are of particular interest because they combine the rigid planarity and extended conjugation of acenes with the chemical stability of oligothiophenes.[5, 6] Unfortunately, the structural rigidity of these materials limits its solubility in common organic solvents, which in turn, restricts their use in the development of solution-processed organic semiconductors. To overcome this problem, oligothienoacenes are frequently functionalized with solubilizing trimethylsilyl (TMS) and triisopropylsilyl (TIPS) substituents, which aid in purification and solution processability.[5-8] However, despite the important role of the silyl groups in the solubility properties of molecular materials for organic electronic applications, only a few studies up to date have investigated the electronic and optical properties and electron transfer characteristics of silyl-functionalized $\pi$-conjugated oligomers.[9-11] On the other hand, even though qualitative interpretations of the well-resolved absorption and emission spectra of oligoacenes can be found in the literature,[12, 13] to the best of our knowledge, a detailed analysis of the vibronic features in silyl end-capped oligothienoacenes has not yet been conducted.

In our previous studies, we analyzed the structural, electrochemical, and optical properties of a series of triisopropylsilyl end-capped oligothienoacenes to gain insight into the fundamental 
structure-property relationships with the addition of each fused thiophene ring. We found that the selective enhancement of a very limited number of Raman scatterings is related to strong vibronic coupling between selected skeletal $v(C=C)$ stretching modes in the $1600-1300 \mathrm{~cm}^{-1}$ range and their respective frontier molecular orbitals.[11] Amplified spontaneous emission was observed in the pentathienoacene derivative doped into polystyrene films indicating that this material is a good candidate for active laser materials.[14] Cyclic voltammetry also reveals the formation of stable radical cations for all these oligothienoacenes, and the formation of dications only for the octamer and the heptamer.[15] The absorption and fluorescence spectra show partially resolved vibronic structures even at room temperature due to the absence of conformational disorder, at low temperature, the absorption spectra of all the series and the emission spectra recorded only for selected systems (i.e., for the pentamer and octamer) show two different vibrational progressions with an energy splitting, corresponding to vibrational modes with energies of $v_{1} \sim 1530 \mathrm{~cm}^{-1}$ and $v_{2} \sim 490 \mathrm{~cm}^{-1}$.[15] In this contribution, we focus on the analysis of the vibronic structure and optical properties of a series of silyl end-capped oligothienoacenes (TIPS-Tn-TIPS, where $\mathrm{n}$ indicates the number of fused thiophene rings) ranging in length from the tetramer to octamer (see Figure 1) by using experimental spectroscopic data and quantum-chemical calculations. UV-Vis absorption and fluorescence emission spectroscopy measurements are used to investigate the spectral profiles of all the series in the ground and first singlet excited state at low $(78 \mathrm{~K})$ and room temperature.

We also employed density functional theory (DFT) and time-dependent DFT (TD-DFT) quantum-mechanical calculations to provide a detailed understanding of the electronic and vibrational interactions and their impact on the characteristics of the absorption and emission 
profiles of silyl end-capped oligothienoacenes. When compared against the non-silylfunctionalized oligothienoacenes, the DFT-calculated energies of the frontier molecular orbitals of the silyl-functionalized oligothienoacenes point to an effective silicon-induced hyperconjugation effect, which is more pronounced in the shorter molecules ( $n=4$ and 5). Our calculations also reveal that the end-capping silyl groups have an almost negligible contribution to the total structural (intramolecular) reorganization energy upon electronic excitation. This finding suggests that although functionalization with TMS or TIPS groups may affect the overall charge carrier characteristics (mainly due to significant changes in the solid state packing induced by the presence of bulky end-capping silyl groups), to a large extent the vibronic coupling properties in silyl end-capped oligothienoacenes are mainly determined by their oligothienyl molecular backbone. Finally, the theoretically estimated vibrational frequencies and vibronic coupling constants of the silyl end-capped molecules are used to simulate their experimental absorption and emission spectral profiles. 

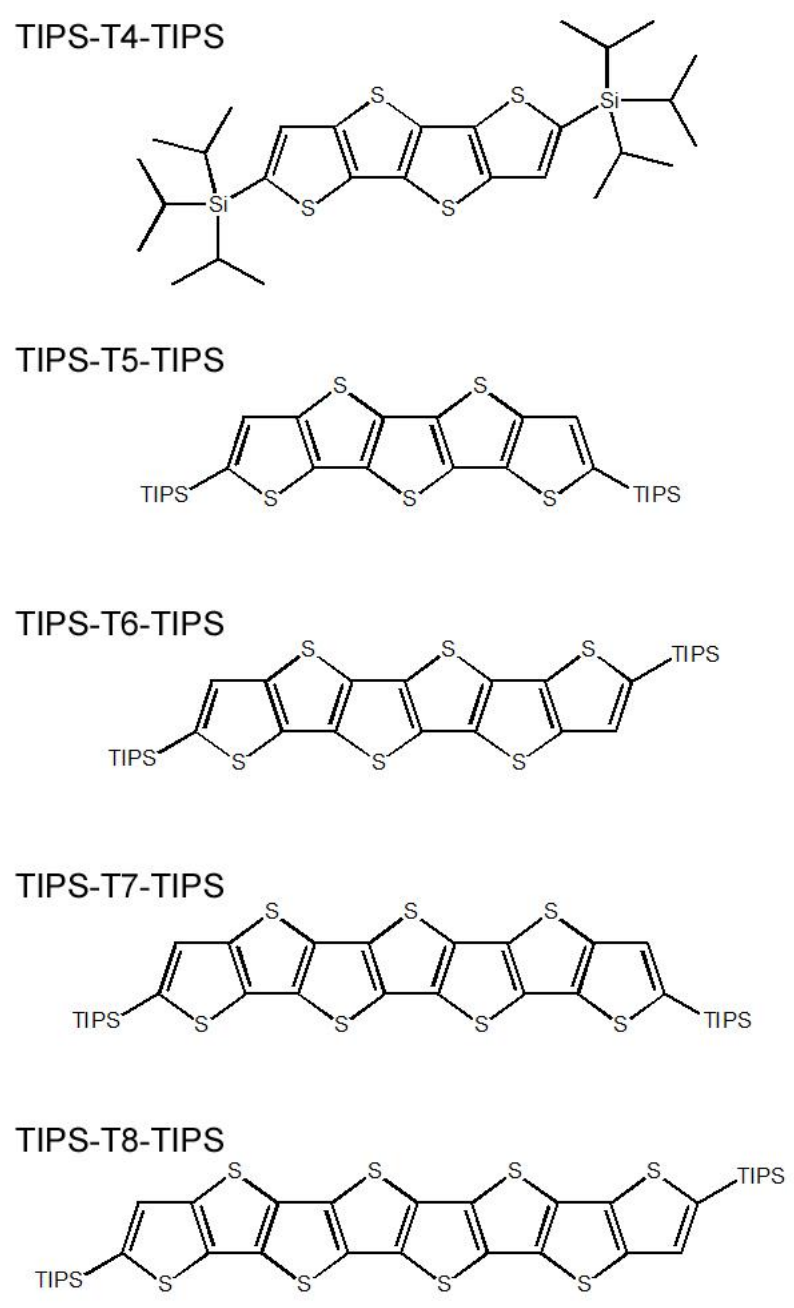

Figure 1. Chemical structures of the series of end-capped oligothienoacenes investigated in this study TIPS-T $n$-TIPS (TIPS: triisopropylsilyl); where $n$ indicates the number of fused thiophene rings. 


\section{Methodology}

\section{A. Experimental}

UV-Vis-NIR absorption spectra were recorded with a diode array Agilent 8453 instrument allowing for fast recording of all electromagnetic absorptions in the 190-1100 nm spectral region. Fluorescence emission spectra were measured using a FL920P spectrometer from Edinburgh Instruments. No fluorescent contaminants were detected upon excitation in the wavelength region of experimental interest. Solutions for emission measurements were prepared with an absorbance in the visible spectral region between 0.1 and 0.2 . 2-Methyltetrahydrofuran (MeTHF) was pre-dried over $\mathrm{KOH}$ for 3 days, filtered, distilled from $\mathrm{CaH}_{2}$, and stored under inert atmosphere. Low-temperature spectra were recorded using an OptistatDN-HT nitrogen bath optical cryostat from Oxford Instruments.

\section{B. Computational and Theoretical Methodology}

Figure 2 represents the potential energy surfaces (PES) for the electronic states $S_{0}$ and $S_{1}$ corresponding to the ground and excited state of the molecule, respectively. The intramolecular reorganization energy $\lambda_{\text {reorg }}$ during an electronic transition consists of two terms related to the geometry relaxation energies upon going from the ground-state geometry to the excited-state geometry and vice-versa,

$$
\lambda_{\text {reorg }}=\lambda_{\text {rel }}^{(1)}+\lambda_{\text {rel }}^{(2)}
$$


Here $\lambda_{\text {rel }}^{(1)}$ and $\lambda_{\text {rel }}^{(2)}$ represent the relaxation of a given molecule as it undergoes $S_{0} \rightarrow S_{1}$ and $S_{1} \rightarrow$ $\mathrm{S}_{0}$ electronic transitions; $\lambda_{\text {rel }}^{(1)}$ is related to the relaxation of a molecular excited state to its excited-state potential energy minimum while $\lambda_{\text {rel }}^{(2)}$ is related to the relaxation of the molecule to its ground-state potential energy minimum after emission. These two energy terms can be computed directly from the adiabatic potential energy surfaces, as schematically indicated in Figure 2 and described in detail, e.g., in References [16, 17].

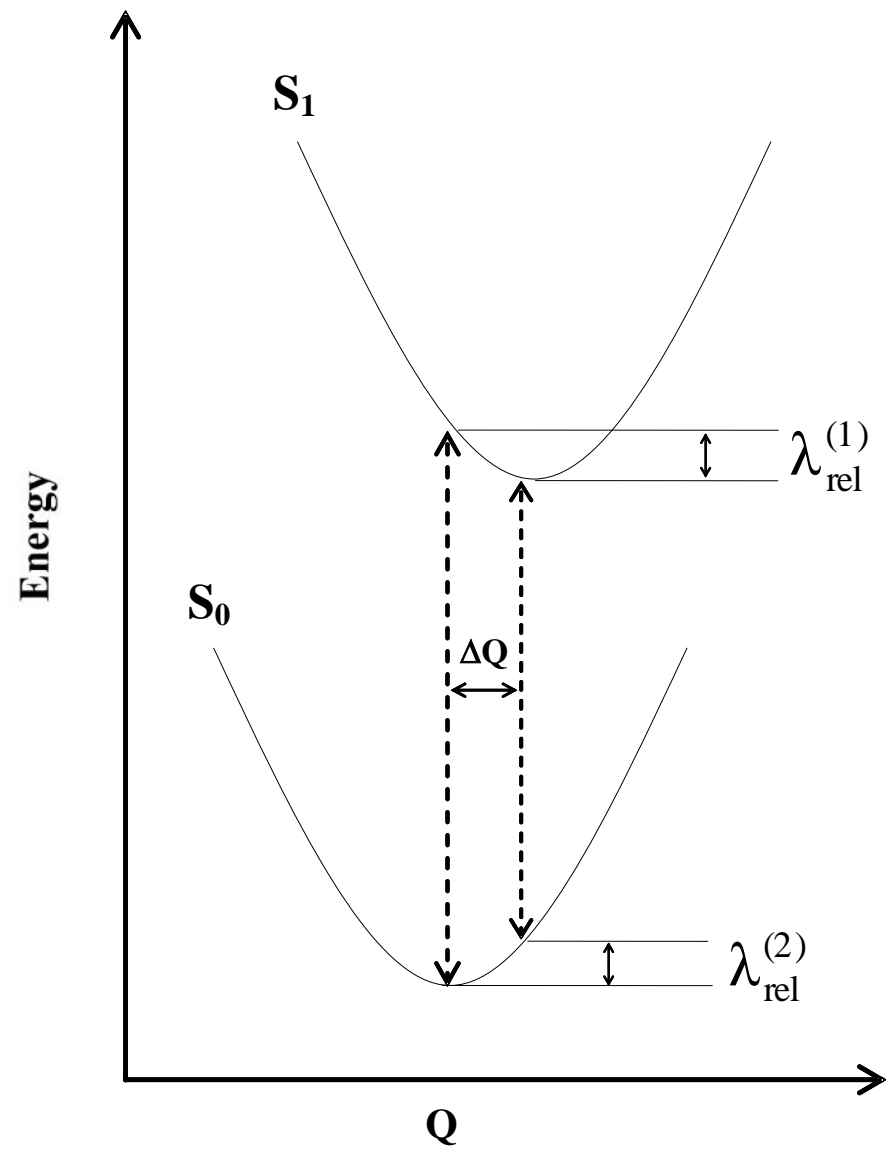


Figure 2. Sketch of the potential energy surfaces for the molecular ground state $S_{0}$ and excited state $S_{1}$, showing the vertical transitions (dashed lines), the normal mode displacement $\Delta \mathrm{Q}$, and the relaxation energies $\lambda_{\text {rel }}^{(1)}$ and $\lambda_{\text {rel }}^{(2)}$.

The contribution of each vibrational mode to $\lambda_{\text {rel }}$ was obtained by expanding the respective potential energy surfaces into a power series of the normal coordinates. In the harmonic approximation, the relaxation energy $\lambda_{\text {rel }}$ is defined as: $[18,19]$

$$
\begin{gathered}
\lambda_{\text {rel }}=\sum \lambda_{\mathrm{i}}=\sum \hbar \omega_{\mathrm{i}} \mathrm{S}_{\mathrm{i}} \\
\lambda_{\mathrm{i}}=\frac{\mathrm{k}_{\mathrm{i}}}{2} \Delta \mathrm{Q}_{\mathrm{i}}^{2}, \quad \mathrm{~S}_{\mathrm{i}}=\frac{\lambda_{\mathrm{i}}}{\hbar \omega_{\mathrm{i}}}
\end{gathered}
$$

Here, the summations run over the vibrational modes; $\Delta \mathrm{Q}_{\mathrm{i}}$ represents the displacement along the normal mode $(\mathrm{NM}) \mathrm{i}$ between the equilibrium positions of the two electronic states of interest; $\mathrm{k}_{\mathrm{i}}$ and $\omega_{\mathrm{i}}$ are the corresponding force constants and vibrational frequencies; $S_{\mathrm{i}}$ denotes the HuangRhys factor (dimensionless electron-vibration coupling constant).

The Huang-Rhys factors $S_{i}$ related to the $S_{0}-S_{1}$ transitions (and vice-versa) were computed using a modified version of the DUSHIN program developed by Reimers.[19] The shapes of the emission and absorption spectra were simulated in the framework of the Born-Oppenheimer and Franck-Condon approximations according to the procedure described in detail elsewhere.[17] In these calculations, the B3LYP frequencies of both the ground and excited state have been scaled, according to the literature, with the same empirical factor of 0.9613.[20] Note also that for the simulation of the emission spectra the cubic frequency dependence was not taken into account; for specific details of this effect on $\pi$-conjugated molecular systems, see for example Reference [21]. The convolution of the resulting spectra was carried out with Gaussian function of uniform 
width, $\sigma_{\mathrm{FWHM}}=0.03 \mathrm{eV}$ for TMS-T $n$-TMS. The onset of the simulated spectra was chosen to match the maximum of the first peak of the simulated spectrum with the maximum of the $(0-0)$ absorption (emission) line found experimentally.

For the sake of computational convenience and efficiency, in all the quantum-chemical calculations the bulky triisopropylsilyl substituents shown in Figure 1 were replaced by trimethylsilyl groups. First, the optimized equilibrium structure and vibrational normal modes of the $S_{0}$ state were obtained using DFT; for the $S_{1}$ state both the geometry optimization and vibrational normal modes calculations were obtained from TD-DFT.[22, 23] Geometry determination at the TD-DFT level was recently shown to reproduce very well the vibronic structures in the absorption spectra of many large organic molecules.[24, 25] In all cases, we employed the B3LYP exchange-correlation functional[26, 27] and the 6-31G(d,p)[28-30] basis set, as implemented in the TURBOMOLE package.[31]

\section{Results and Discussion}

\section{A. Experimental Spectra}

Figure 3 displays the normalized UV-Vis absorption and fluorescence spectra of TIPS-T $n$-TIPS recorded at low $(78 \mathrm{~K})$ and room temperature. Table 1 shows the wavelengths of the absorption and fluorescence emission maxima recorded at both temperatures. 

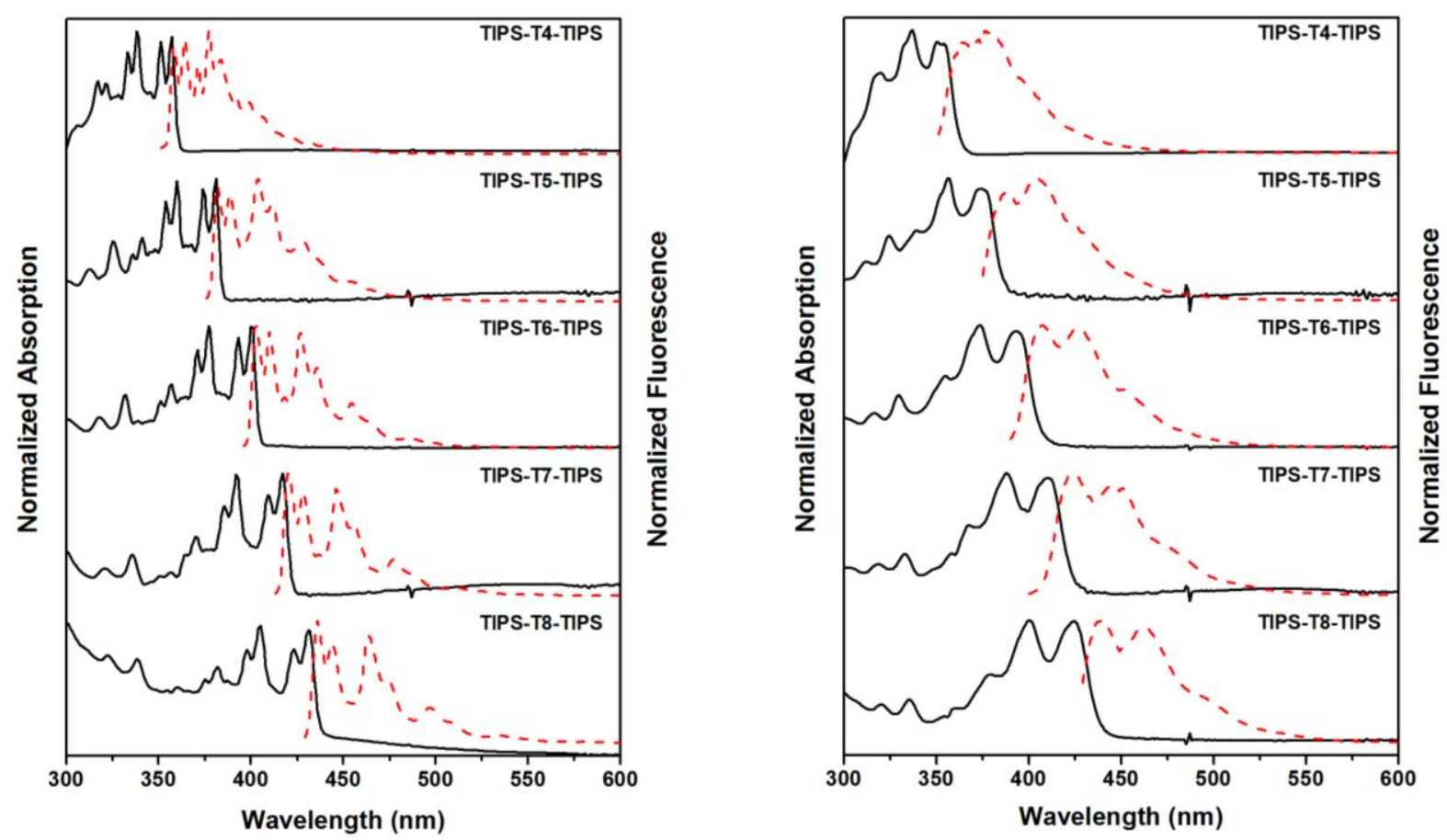

Figure 3. Normalized UV-Vis absorption (solid lines) and fluorescence emission (dashed lines) spectra of TIPS-Tn-TIPS oligothienoacenes in MeTHF recorded at $78 \mathrm{~K}$ (left) and at room temperature (right).

Table 1. UV-Vis absorption and fluorescence emission maxima ( $\lambda_{\max }$ in $\mathrm{nm}$ ) observed for TIPS$\mathrm{T} n$-TIPS at room temperature and low temperature (values given between parentheses), molar absorption coefficient $(\log \varepsilon)$ and fluorescence quantum yield $\left(\phi_{\mathrm{F}}\right)$.

\begin{tabular}{ccccc}
\hline \multirow{2}{*}{ Compound } & \multicolumn{2}{c}{ Absorption } & \multicolumn{2}{c}{ Emission } \\
& $\lambda_{\max }^{\mathrm{a}}$ & $\log \varepsilon$ & $\lambda_{\max }$ & $\phi_{\mathrm{F}}$ \\
\hline TIPS-T4-TIPS & $350^{\mathrm{b}}(357-351)^{\mathrm{b}}$ & $4.52^{\mathrm{d}}$ & $363^{\mathrm{b}}(358-364)^{\mathrm{c}}$ & $0.08^{\mathrm{d}}$ \\
TIPS-T5-TIPS & $375^{\mathrm{b}}(381-374)^{\mathrm{b}}$ & $4.56^{\mathrm{e}}$ & $387^{\mathrm{b}}(382-389)^{\mathrm{c}}$ & $0.14^{\mathrm{c}}$ \\
TIPS-T6-TIPS & $393^{\mathrm{b}}(400-393)^{\mathrm{b}}$ & $4.68^{\mathrm{d}}$ & $408^{\mathrm{b}}(403-410)^{\mathrm{c}}$ & $0.32^{\mathrm{d}}$ \\
TIPS-T7-TIPS & $409^{\mathrm{b}}(417-409)^{\mathrm{b}}$ & $4.86^{\mathrm{f}}$ & $424^{\mathrm{b}}(420-428)^{\mathrm{c}}$ & $0.41^{\mathrm{c}}$ \\
TIPS-T8-TIPS & $425^{\mathrm{b}}(431-423)^{\mathrm{b}}$ & $4.76^{\mathrm{d}}$ & $440^{\mathrm{b}}(436-444)^{\mathrm{c}}$ & $0.39^{\mathrm{d}}$ \\
\hline
\end{tabular}


${ }^{\mathrm{a}}$ In THF.

${ }^{\mathrm{b}}$ From Reference [15].

${ }^{\mathrm{c}}$ From this work.

${ }^{\mathrm{d}}$ In o-dichlorobenzene, from Reference [5].

${ }^{\mathrm{e}}$ In THF, from Reference [32].

${ }^{\mathrm{f}}$ In $\mathrm{CH}_{2} \mathrm{Cl}_{2}$, from Reference [6].

SS

At room temperature, the normalized absorption and fluorescence emission spectra of all the TIPS-T $n$-TIPS oligomers show a broad spectrum with partially resolved vibronic structures in good agreement with our previous data.[14, 15] The appearance of vibrational fine structure in the absorption spectra in solution is a fairly unique property of oligothienoacenes, which is generally not observed in non-fused $\alpha$-oligothiophenes.[33] This, in principle, is attributed to the rigidity of the fully-fused thiophene backbone, which drastically reduces the conformational disorder. In our previous work,[15] the single excited electronic states of all these oligomers were calculated at the TD-DFT level. In good agreement with the experimental data, the existence of one strong electronic transition in the near-UV-Visible range which undergoes a large bathochromic shift as the oligomer lengthens is predicted. This transition implies the excitation to the first excited state $\mathrm{S} 1$, which is shown to increase in intensity with the oligomer length (i.e., larger $f$ values). This agrees with the slight increase in the molar absorption coefficient. The fluorescence spectrum recorded at room temperature is also structured; a quite small shift is observed between the corresponding first peaks in UV-Vis absorption and photoluminescence spectrum, which becomes smaller as the number of thiophene rings increases. As the $\pi$-conjugation is extended, the fluorescence quantum yield increases and the emission maximum shifts to longer wavelengths (i.e., from $363 \mathrm{~nm}$ and $\phi_{\mathrm{F}}=0.08$ in TIPS-T4TIPS to $440 \mathrm{~nm}$ and $\phi_{\mathrm{F}}=0.39$ in TIPS- T8-TIPS, see Table 1). 
In agreement with our previous data,[15] the vibronic peaks in the UV-Vis and fluorescence spectra of TIPS-Tn-TIPS sharpen and become stronger upon cooling (see Figure 3). The lowestenergy optical absorption clearly shows two different vibronic progressions with different energy splitting, for which up to three replicas are seen. The vibronic progression corresponds to the vibrational modes having frequencies of $v_{1} \sim 1530 \mathrm{~cm}^{-1}$ and $v_{2} \sim 490 \mathrm{~cm}^{-1}$. Two well-resolved vibronic progressions are also observed in the fluorescence spectrum at low temperature $\left(v_{1} \sim\right.$ $1400 \mathrm{~cm}^{-1}$ and $v_{2} \sim 450 \mathrm{~cm}^{-1}$ ); while, a moderate energy-splitting is observed. In comparison to the UV-Vis absorption spectrum, the energy-splitting is smaller. The changes accompanying cooling can be interpreted as a consequence of a reduction in the inhomogeneous broadening induced by the considerable variation of local environments (i.e., the spectra of the individual molecules at low temperature solids are much similar than in solution because of the loss of some conformational disorder).[34-36] The resolution of the vibrational structure at low temperature is less pronounced for the longer oligomers (i.e., hexamer, heptamer and octamer) due to the fact that the coupling with low-frequency vibrations ca. $100 \mathrm{~cm}^{-1}$ becomes more significant, as discussed below in more detail in section D

Finally, we discuss the experimentally observed red-shift signatures of the UV-Vis absorption peaks of the silyl end-capped oligothienoacenes as compared against their non-silylfunctionalized counterparts. This analysis was carried out by looking at the DFT-calculated energies (and topologies) of the molecular orbitals involved in the $S_{0} \rightarrow S_{1}$ electronic transition of both sets of molecules. For a complete list of the UV-Vis absorption peaks in unsubstituted oligothienoacenes see Table S1. 
Silicon substituents are known to induce an effective hyperconjugation when coupled with carbon atoms.[37] In the case of organic semiconductors, this effect has been demonstrated both experimentally and theoretically for various silicon-containing $\pi$-conjugated organic systems,[38, 39] for which, the $\pi^{*}$-orbital of the aromatic system mixes effectively with the exocyclic Si-C $\sigma^{*}$-orbital to afford a low-lying lowest unoccupied molecular orbital (LUMO) and a relatively small band gap. In principle, a similar stabilizing effect is also expected for the case of the highest occupied molecular orbital (HOMO) in $\pi$-conjugated systems.[40, 41] However, as recently observed by $\mathrm{Lu}$ et al.,[40] the introduction of thiophene rings into the molecular core of silicon-containing semiconducting materials leads to an energetic destabilization of the HOMOs and stabilization of the LUMOs.

When compared against their non-silyl-functionalized counterparts, the silyl end-capped oligothienoacenes (except for the LUMO of TMS-T8-TMS) show the above-mentioned stabilization [destabilization] of the LUMO [HOMO] energy levels, as a result of an effective silicon-induced hyperconjugation effect (see Figure 4). The energy difference between the LUMOs in the 8-ring systems is approximately $1 \mathrm{meV}$, which is too small to change the overall (observed) LUMO stabilization trend of the TMS-Tn-TMS oligomers. This effective siliconinduced hyperconjugation contributes then to the systematic reduction of the calculated HOMOLUMO gaps and to the observed red-shift of the UV-Vis absorption peaks in silicon-containing oligothienoacenes versus those measured in their unsubstituted counterparts. As an example of the silicon-induced effect, in Figure S1, we reported the UV-Vis absorption and emission profiles of the pentathienoacene and TMS-T5-TMS molecules. 


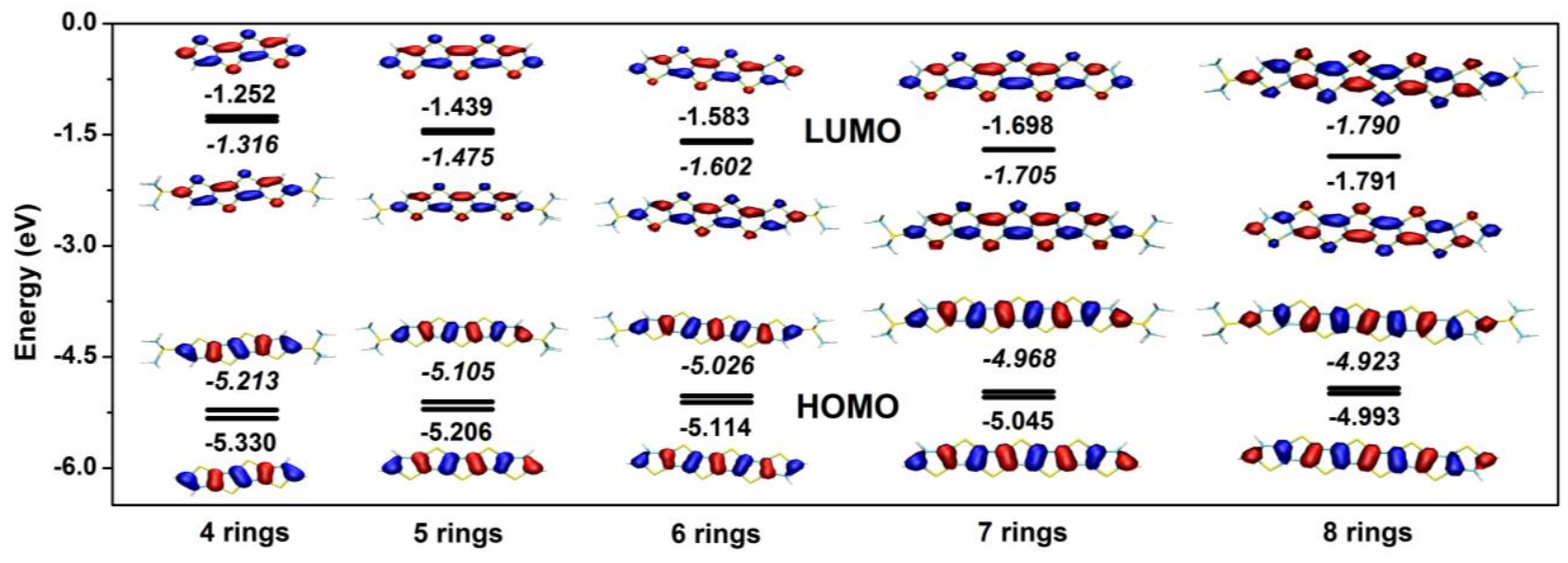

Figure 4. DFT/B3LYP/6-31G** energy levels around the HOMO-LUMO gap region and molecular orbital topologies of the HOMO and LUMO of tetra(penta, hexa, hepta, and octa)thienoacene and TMS-T4(5, 6, 7, and 8)-TMS. The italicized energy values correspond to those of the TMS-T $n$-TMS oligomers.

\section{B. Ground and Excited Structures}

We investigate here the geometries of the ground state $\left(\mathrm{S}_{0}\right)$ and first excited state $\left(\mathrm{S}_{1}\right)$ obtained from DFT and TD-DFT calculations. The calculated bond lengths changes observed upon excitation for TMS-T4-TMS and TMS-T8-TMS are summarized in Table 2 (see Table S2 in the Supporting Information for the data of the rest of the compounds.) The degree of aromatization/quinoidization of the conjugated backbone can be easily quantified by using the $\mathrm{C}-\mathrm{C}$ bond-length alternation (BLA) parameter. The BLA parameter is computed for each thiophene ring as the difference between the length of the $C_{\beta}-C_{\beta}$ and the average of the two $\mathrm{C}_{\alpha}=\mathrm{C}_{\beta}$ bonds. An aromatic ring is thus characterized by a positive BLA value, while a quinoidlike ring shows a negative BLA value. Table 3 collects the BLA values obtained for TMS-T $n$ TMS oligomers. In the ground state, the thiophene rings display positive BLA values indicating that their molecular structure is of aromatic-type, in agreement with previous findings.[7, 15] 
The geometry changes upon excitation from the $S_{0}$ state into the optimized $S_{1}$ geometry correspond to an elongation of the $\mathrm{C}_{\alpha}=\mathrm{C}_{\beta}$ double bonds and a shortening of the $\mathrm{C}_{\beta}-\mathrm{C}_{\beta}$ single bonds, i.e., a switch in bond length alternation. These $\mathrm{C}-\mathrm{C}$ bond length changes are found to be more pronounced toward the molecular center (i.e., the BLA values computed for TMS-T8-TMS changes from $0.034 / 0.023 / 0.021 / 0.020 \AA$ in the $\mathrm{S}_{0}$ state to $0.008 /-0.019 /-0.034 /-0.042 \AA$ in the $\mathrm{S}_{1}$ state, in going from the outer to the center rings). The change in sign in the BLA values clearly illustrates the quinoidization of the conjugated carbon skeleton. On the other hand, the $\mathrm{C}-\mathrm{S}$ bond lengths are found to increase on going from $S_{0}$ to $S_{1}$, with a larger impact on the inner than on the outer rings. It is interesting to note that the geometrical changes upon electronic excitation are less pronounced in the longer oligomers in comparsion with the shorter ones; for instance, the $\mathrm{C}-\mathrm{C}$ bond length changes upon excitation are in the range of $-0.039 / 0.048 \AA$ in TMS-T4-TMS while they are in the range of $-0.030 / 0.025 \AA$ in TMS-T8-TMS (see Table 2 for TMS-T4-TMS and TMS-T8-TMS and Table S2 for the rest of compounds). 
Table 2. Selected bond lengths $(\AA)$ in the ground-state and excited state of TMS-Tn-TMS molecules ( $n=4$ and 8 ) as determined by DFT/TD-DFT calculations.
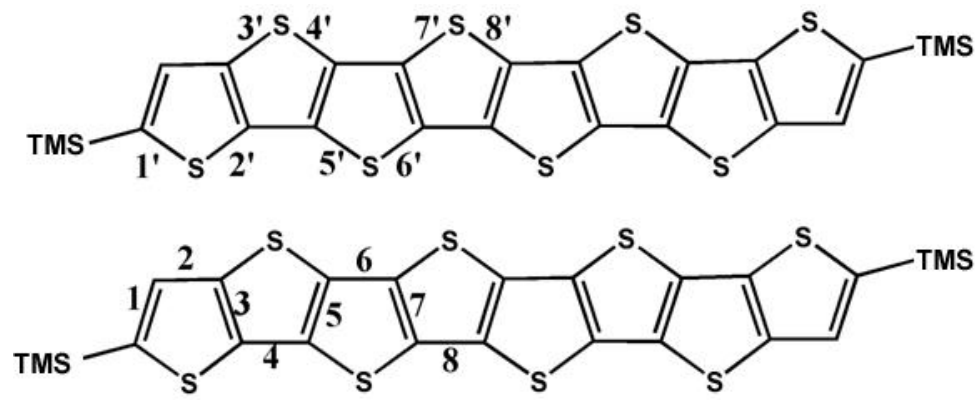

\begin{tabular}{cccccccc}
\hline & \multicolumn{3}{c}{ TMS-T4-TMS } & & \multicolumn{3}{c}{ TMS-T8-TMS } \\
\cline { 2 - 3 } \cline { 6 - 7 } Bonds & $\mathrm{S}_{0}$ & $\mathrm{~S}_{1}$ & $\Delta\left(\mathrm{S}_{1}-\mathrm{S}_{0}\right)$ & & $\mathrm{S}_{0}$ & $\mathrm{~S}_{1}$ & $\Delta\left(\mathrm{S}_{1}-\mathrm{S}_{0}\right)$ \\
\hline $\mathbf{C}-\mathbf{C}$ & & & & & & \\
$\mathbf{1}$ & 1.379 & 1.405 & 0.026 & & 1.379 & 1.390 & 0.011 \\
$\mathbf{2}$ & 1.421 & 1.394 & -0.027 & & 1.421 & 1.408 & -0.013 \\
$\mathbf{3}$ & 1.394 & 1.430 & 0.036 & & 1.395 & 1.411 & 0.016 \\
$\mathbf{4}$ & 1.421 & 1.382 & -0.039 & & 1.419 & 1.398 & -0.021 \\
$\mathbf{5}$ & 1.396 & 1.444 & 0.048 & & 1.397 & 1.422 & 0.025 \\
$\mathbf{6}$ & 1.421 & 1.382 & -0.039 & & 1.418 & 1.391 & -0.027 \\
$\mathbf{7}$ & 1.394 & 1.430 & 0.036 & & 1.397 & 1.428 & 0.031 \\
$\mathbf{8}$ & 1.421 & 1.394 & -0.027 & & 1.417 & 1.387 & -0.030 \\
$\mathbf{C - S}$ & & & & & & \\
$\mathbf{1}$ & 1.770 & 1.778 & 0.008 & & 1.770 & 1.773 & 0.003 \\
$\mathbf{2}$, & 1.738 & 1.758 & 0.020 & & 1.738 & 1.746 & 0.008 \\
$\mathbf{3}$, & 1.761 & 1.779 & 0.018 & & 1.762 & 1.768 & 0.006 \\
$\mathbf{4}$, & 1.756 & 1.771 & 0.015 & & 1.755 & 1.764 & 0.009 \\
$\mathbf{5}$, & 1.756 & 1.771 & 0.015 & & 1.756 & 1.764 & 0.008 \\
$\mathbf{6}$, & 1.761 & 1.779 & 0.018 & & 1.755 & 1.766 & 0.011 \\
$\mathbf{7}$, & 1.738 & 1.758 & 0.020 & & 1.756 & 1.767 & 0.011 \\
$\mathbf{8}$, & 1.770 & 1.778 & 0.008 & & 1.756 & 1.768 & 0.012 \\
\hline
\end{tabular}


Table 3. BLA values (in $\AA$ ) calculated for thiophene rings of TMS-Tn-TMS molecules in their ground state $\left(\mathrm{S}_{0}\right)$ and excited state $\left(\mathrm{S}_{1}\right)$.

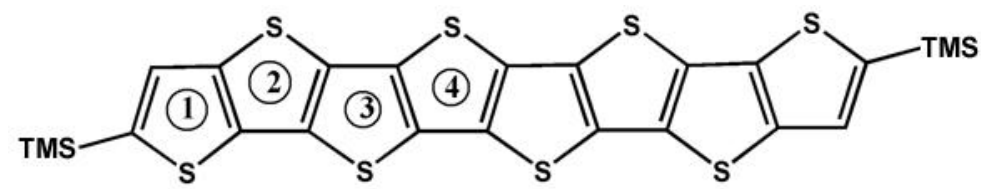

\begin{tabular}{|c|c|c|c|c|c|c|c|c|c|c|}
\hline \multirow[b]{2}{*}{ Ring } & \multicolumn{2}{|c|}{ TMS-T4-TMS } & \multicolumn{2}{|c|}{ TMS-T5-TMS } & \multicolumn{2}{|c|}{ TMS-T6-TMS } & \multicolumn{2}{|c|}{ TMS-T7-TMS } & \multicolumn{2}{|c|}{ TMS-T8-TMS } \\
\hline & $\mathrm{S}_{0}$ & $S_{1}$ & $\mathrm{~S}_{0}$ & $\mathrm{~S}_{1}$ & $\mathrm{~S}_{0}$ & $S_{1}$ & $\mathrm{~S}_{0}$ & $\mathrm{~S}_{1}$ & $\mathrm{~S}_{0}$ & $\mathrm{~S}_{1}$ \\
\hline 1 & 0.035 & -0.024 & 0.035 & -0.012 & 0.034 & -0.004 & 0.035 & 0.003 & 0.034 & 0.008 \\
\hline 2 & 0.026 & -0.053 & 0.025 & -0.043 & 0.025 & -0.033 & 0.023 & -0.025 & 0.023 & -0.019 \\
\hline 3 & 0.026 & -0.053 & 0.023 & -0.055 & 0.023 & -0.049 & 0.021 & -0.041 & 0.021 & -0.034 \\
\hline 4 & 0.035 & -0.024 & 0.025 & -0.043 & 0.023 & -0.049 & 0.021 & -0.046 & 0.020 & -0.042 \\
\hline
\end{tabular}

\section{Reorganization Energy}

The intramolecular relaxation energies $\lambda_{\text {rel }}$ related to the $S_{0} \rightarrow S_{1}$ and $S_{1} \rightarrow S_{0}$ transitions for the series of silyl end-capped oligothienoacenes were obtained from potential energy surfaces calculations and by partitioning the intramolecular relaxation energy into the contribution of each normal mode according to Equations 2 and 3. The calculated values related to the $S_{0} \rightarrow S_{1}$ and $S_{1}$

$\rightarrow \mathrm{S}_{0}$ transitions are listed in Table 4. Overall, we found a good agreement between the potential energy surface calculations and the normal-mode approach. The results also show a monotonical reduction of the relaxation energies with increase of the number of thiophene units in the oligomeric chain of the TMS-Tn-TMS oligothienoacenes. This observation is in good agreement with previous quantum-chemical studies of various aromatic-based chemical structures.[7, 16, 42, 43]

Also, we observed that along the series of end-capped oligothienoacenes there are only a couple of normal modes that mainly determine the vibronic progressions in spectra of these molecules 
(vibrational modes with energies of $v_{1} \sim 1500 \mathrm{~cm}^{-1}$ and $v_{2} \sim 500 \mathrm{~cm}^{-1}$ ). The qualitative characteristics of these vibrations are very similar in both electronic transitions. Thus, one could expect the UV-Vis absorption and fluorescence spectra to be near-perfect mirror images; which, indeed, is the case for the series of TMS-Tn-TMS oligothienoacenes. However, note that the spacing of the vibrational features is slightly larger in absorption than in emission; this agrees with the slightly larger Huang-Rhys factors (see Table 5 and Table S3) found for the lowfrequency modes (below $600 \mathrm{~cm}^{-1}$ ) in the $S_{0} \rightarrow S_{1}$ transition versus those observed for the $S_{1} \rightarrow$ $\mathrm{S}_{0}$ transition. Additionally, the difference in spacing of the vibronic progression peaks in the spectroscopic profiles of these molecular structures is consistent with the widely accepted view that aromatic-like compounds assume a quinoidal form in the electronic excited state. For example, we note that the excited-state properties of the silyl end-capped oligothienoacenes show the typical alternation of single and double carbon-carbon bonds and the characteristic shift of the ring $\mathrm{C}-\mathrm{C}$ stretching mode toward the high-frequency region of the vibronic spectrum.[44, 45] The change in sign of the BLA values reported in Table 3 (from positive to negative) is also in good agreement with the above arguments.

Table 4. Total relaxation energy $\lambda_{\text {rel }}$ of the $S_{0} \rightarrow S_{1}$ and $S_{1} \rightarrow S_{0}$ transitions of TMS-Tn-TMS obtained from potential energy surfaces (PES) and normal-mode (NM) calculations.

\begin{tabular}{ccccc}
\hline & \multicolumn{2}{c}{$\mathrm{S}_{0} \rightarrow \mathrm{S}_{1}$ transition } & \multicolumn{2}{c}{$\mathrm{S}_{1} \rightarrow \mathrm{S}_{0}$ transition } \\
compound & $\lambda_{\text {rel }}(\mathrm{PES}) / \mathrm{meV}$ & $\lambda_{\text {rel }}(\mathrm{NM}) / \mathrm{meV}$ & $\lambda_{\text {rel }}(\mathrm{PES}) / \mathrm{meV}$ & $\lambda_{\text {rel }}(\mathrm{NM}) / \mathrm{meV}$ \\
\hline TMS-T4-TMS & 207 & 215 & 226 & 220 \\
TMS-T5-TMS & 192 & 198 & 210 & 206 \\
TMS-T6-TMS & 180 & 185 & 198 & 195 \\
TMS-T7-TMS & 171 & 175 & 189 & 186 \\
TMS-T8-TMS & 164 & 168 & 181 & 179 \\
\hline
\end{tabular}


The decomposition of the value of the $\lambda_{\text {rel }}$ into contributions of each normal-mode for the TMSTn-TMS oligothienoacenes permits an assignment of the contributions of the low- and highfrequency vibrations to the total value of the calculated relaxation energies. We found that for both electronic transitions approximately $70 \%-75 \%$ of the relaxation energy comes from highfrequency vibrations in the range of $1200-1600 \mathrm{~cm}^{-1}$; which include a particularly dominant vibration characterized by a $\mathrm{C}-\mathrm{C}$ stretching of the inner-most rings (see Figure 5). On the other hand, the contributions of low-frequency vibrations (below $600 \mathrm{~cm}^{-1}$ ) account for only $25 \%$ $30 \%$ of the total value of $\lambda_{\text {rel }}$ and include vibrations that are near to $500 \mathrm{~cm}^{-1}$ and characterized by stretches along the short molecular axis (i.e., via the $\mathrm{C}-\mathrm{S}-\mathrm{C}$ atoms of the thiophene rings). As an illustrative example of decomposition of the $\lambda_{\text {rel }}$ into the normal-mode contributions, the TDDFT-derived (Huang-Rhys factors, $S$ ) related to the $S_{0} \rightarrow S_{1}$ and $S_{1} \rightarrow S_{0}$ transitions in TMS-T5TMS are shown in Table 5 (see Supporting Information for the corresponding data of the other molecular structures investigated in this study). 
TMS-T5-TMS

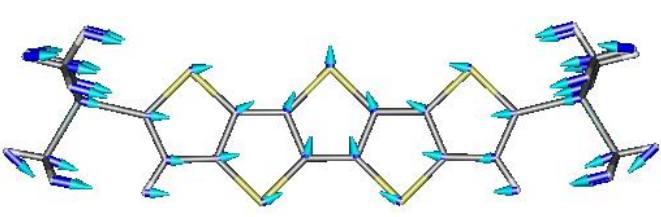

$\omega=138 \mathrm{~cm}^{-1}$

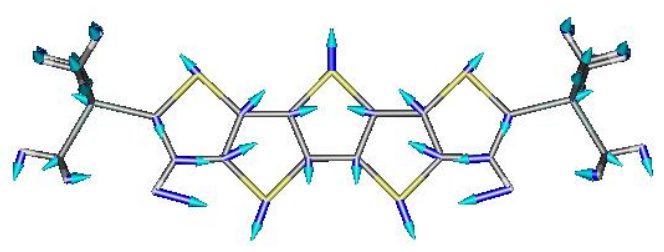

$\omega=499 \mathrm{~cm}^{-1}$

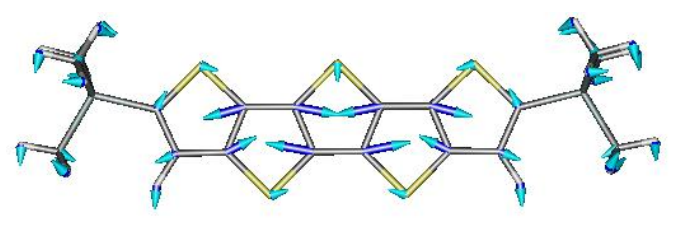

$\omega=1482 \mathrm{~cm}^{-1}$
TMS-T8-TMS

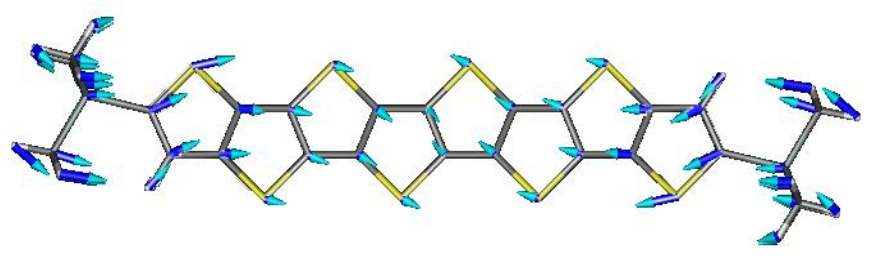

$\omega=114 \mathrm{~cm}^{-1}$

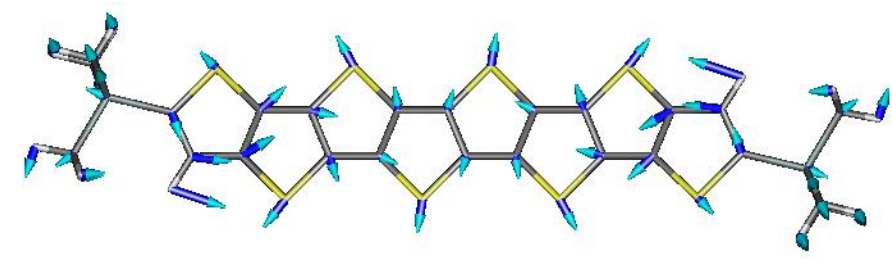

$\omega=502 \mathrm{~cm}^{-1}$

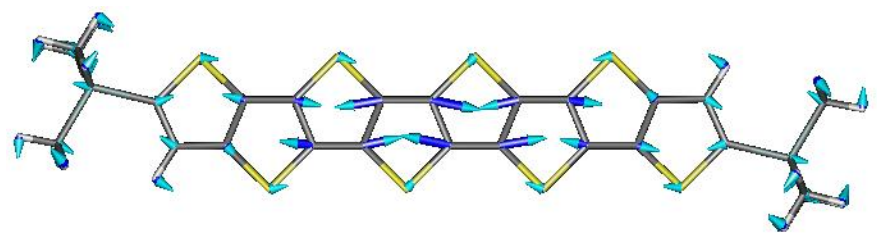

$\omega=1476 \mathrm{~cm}^{-1}$

\section{Transition Electron Densities}
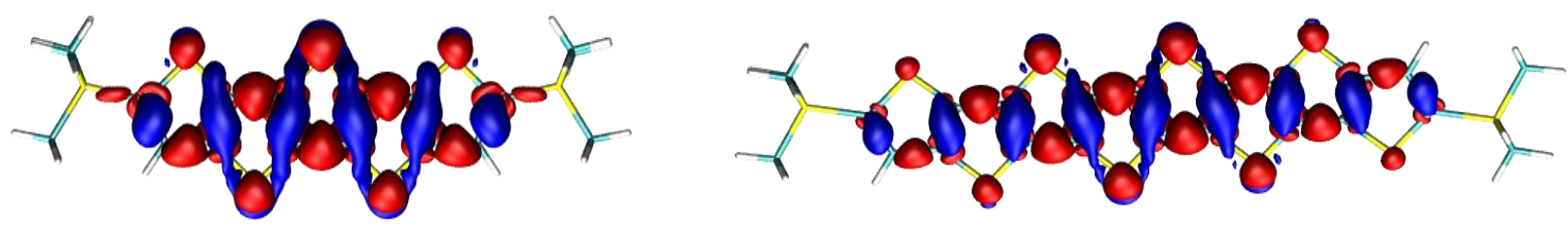

Figure 5. (top) Illustrations of the normal modes yielding the largest Huang-Rhys factors and (bottom) electron density change in the S0-S1 electronic transition of TMS-T5-TMS and TMST8-TMS (left and right, respectively). The low-frequency modes involve mostly the sulfur atoms, whereas the high-frequency modes involve the carbon atoms. 
Table 5. DFT/TD-DFT/B3LYP/6-31G** estimates of frequencies $\omega$, Huang-Rhys factors $\mathrm{S}$, and relaxation energies $\lambda_{\text {rel }}$ related to $\mathrm{S}_{0} \rightarrow \mathrm{S}_{1}$ and $\mathrm{S}_{1} \rightarrow \mathrm{S}_{0}$ transitions of TMS-T5-TMS (only the most strongly coupled vibrations are shown).

\begin{tabular}{cccccc}
\hline & $\mathrm{S}_{0} \rightarrow \mathrm{S}_{1}$ transition & \multicolumn{3}{c}{$\mathrm{S}_{1} \rightarrow \mathrm{S}_{0}$ transition } \\
$\omega\left(\mathrm{cm}^{-1}\right)$ & $\mathrm{S}$ & $\lambda_{\text {rel }}(\mathrm{eV})$ & $\omega\left(\mathrm{cm}^{-1}\right)$ & $\mathrm{S}$ & $\lambda_{\text {rel }}(\mathrm{eV})$ \\
\hline 41 & 0.037 & 0.000 & 41 & 0.037 & 0.000 \\
117 & 0.039 & 0.001 & 116 & 0.041 & 0.001 \\
138 & 0.122 & 0.002 & 137 & 0.124 & 0.002 \\
240 & 0.138 & 0.004 & 240 & 0.142 & 0.004 \\
264 & 0.030 & 0.001 & 264 & 0.026 & 0.001 \\
478 & 0.142 & 0.008 & 470 & 0.433 & 0.025 \\
499 & 0.608 & 0.038 & 485 & 0.279 & 0.017 \\
1290 & 0.146 & 0.023 & 606 & 0.020 & 0.002 \\
1482 & 0.567 & 0.104 & 1229 & 0.145 & 0.022 \\
1520 & 0.048 & 0.009 & 1578 & 0.628 & 0.123 \\
\hline
\end{tabular}

In Figure 6, we present the calculated vibrational coupling (Huang-Rhys factors, S) as obtained from TD-DFT for the complete series of TMS-Tn-TMS compounds. The different plots of Figure 6 show consistent vibronic-coupling interactions with both low- and high-frequency vibrations. Further inspection of the most-strongly coupled vibrations shows that the main distortions occur within the molecular backbone (i.e., the fused-thiophene rings) of the TMScapped compounds. However, we also note that the vibronic-coupling interactions of the larger end-capped oligothienoacenes (TMS-T6-TMS, TMS-T7-TMS, and TMS-T8-TMS) involve a low-frequency vibration at around $100 \mathrm{~cm}^{-1}$. This vibration (shown in Figure 5) involves, for the case of TMS-T8-TMS, distortions of the silyl-based groups accompanied by lateral distortions of the outer-most thiophene rings; whereas for TMS-T5-TMS, this vibration mainly distorts the atomic positions of the silyl groups. This normal mode posses a large Huang-Rhys factor (i.e., 0.411 for the $114 \mathrm{~cm}^{-1}$ mode of TMS-T8-TMS, see Table S3 in the Supporting Information) and therefore has an effect in the absorption and emission spectra. As discussed in section D, this low-frequency vibration leads to a peak broadening of the absorption and emission profiles of the 
larger end-capped oligothienoacenes, for which the extended motions of the outer-most rings distort significantly the transition electron densities as shown in Figure 5 for TMS-T5-TMS and TMS-T8-TMS (see Figure S2 in the Supporting information for the data of the rest of the compounds). 

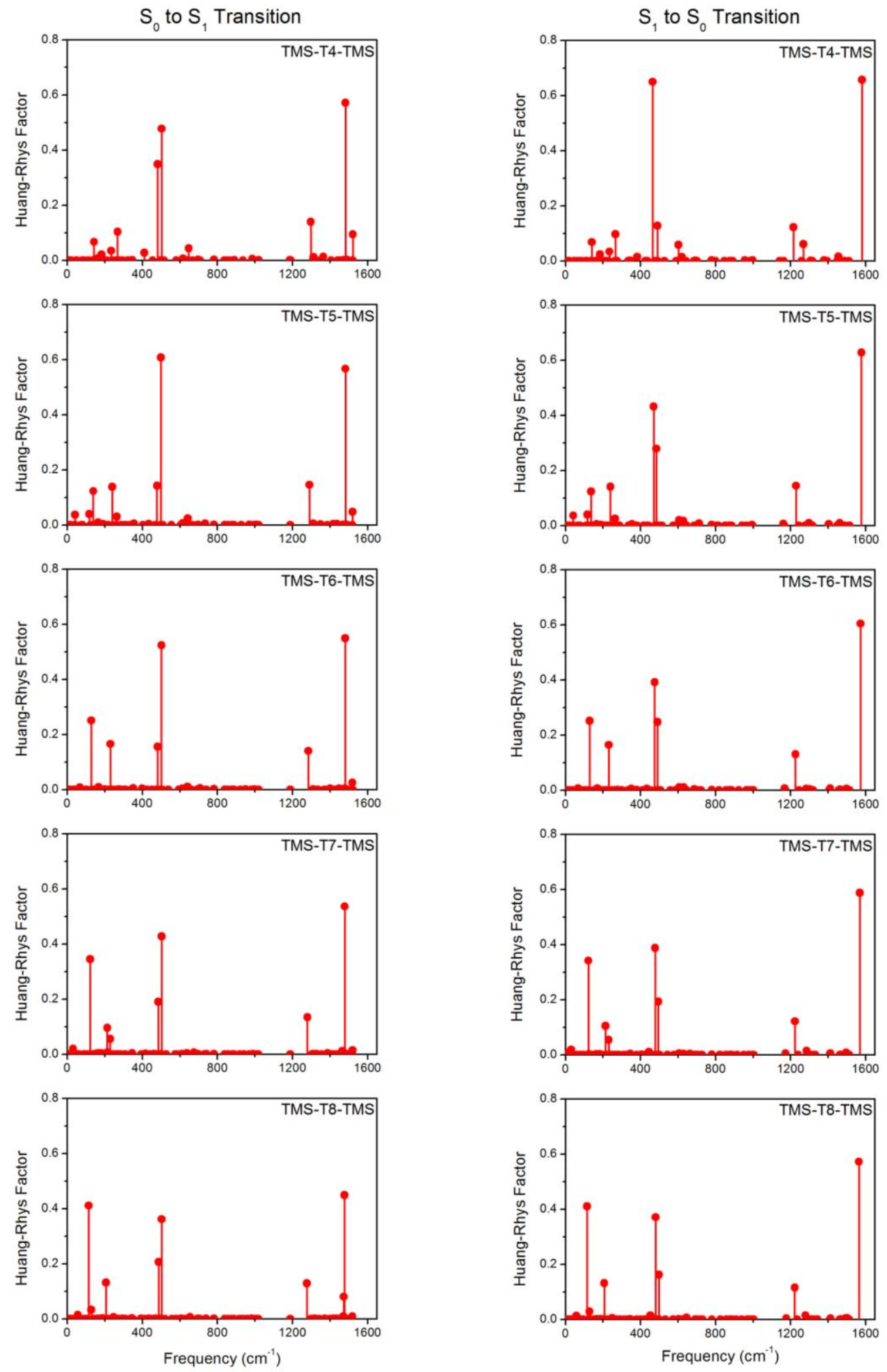

Figure 6. DFT/TD-DFT/B3LYP/6-31G** Huang-Rhys factors related to the $S_{0} \rightarrow S_{1}$ and $S_{1} \rightarrow$ $\mathrm{S}_{0}$ transitions of TMS-Tn-TMS as a function of the normal-mode frequency. 
Finally, to investigate the effective contributions of the silyl-groups to the overall intramolecular reorganization energy $\lambda_{\text {reorg, }}$, we fixed the motions of the oligothienoacene molecular core in the series of TMS-Tn-TMS oligomers, and computed again their respective $\lambda_{\text {reorg }}$ values. We found that the contributions of TMS groups to the total value of $\lambda_{\text {reorg }}$ upon an electronic excitation from the $S_{0} \rightarrow S_{1}$ state and vice-versa, is on the order of $1 \%$ (Table 6).

Table 6. Individual contributions of the oligothienoacene core and of the TMS substituents to the total intramolecular reorganization energy $\left(\lambda_{\text {reorg }}\right)$ in the TMS-T $n$-TMS oligomers.

\begin{tabular}{cccc}
\hline molecule & $\begin{array}{c}\text { TMS }^{\text {a }} \\
\lambda_{\text {reorg }}(\mathrm{meV})\end{array}$ & $\begin{array}{c}\text { oligothienoacene core, }^{\text {b }} \\
\lambda_{\text {reorg }}(\mathrm{meV})\end{array}$ & $\begin{array}{c}\text { total, } \\
\lambda_{\text {reorg }}(\mathrm{meV})\end{array}$ \\
\hline TMS-T4-TMS & 5 & 427 & 432 \\
TMS-T5-TMS & 3 & 395 & 398 \\
TMS-T6-TMS & 1 & 370 & 371 \\
TMS-T7-TMS & 1 & 352 & 353 \\
TMS-T8-TMS & 1 & 337 & 338 \\
\hline
\end{tabular}

${ }^{a}$ The atomic coordinates of the oligothienoacene core were maintained fixed at the $\mathrm{S}_{0}$ geometry positions during the optimization of the $\mathrm{S}_{1}$ state. ${ }^{\mathrm{b}}$ The atomic coordinates of the TMS substituents were constrained at the $S_{0}$ geometry positions during the optimization of the $S_{1}$ state.

\section{Simulation of the UV-Vis absorption and fluorescence}

We employed the results of the normal-mode analysis to simulate the shape of the absorption and emission profiles of the TMS-T $n$-TMS molecular compounds. Only vibrational modes with Huang-Rhys factors larger than 0.05 were used in these simulations since modes with smaller Huang-Rhys factors essentially do not contribute to the description of the electronic transitions of these almost-rigid molecular structures. The results of the simulation are presented in Figure 7. The vibronic features of the experimental and theoretical profiles are in good agreement; highlighting the reliability of the TD-DFT-derived vibronic coupling constants and relaxation 
energies. However, the experimental intensity distributions cannot be perfectly reproduced by the simulated spectra; the calculated intensities of the (0-1) and (0-2) transitions are smaller with respect to the $(0-0)$ peak than those observed experimentally. This underestimation is likely related to the amount of exact Hartree-Fock exchange in the B3LYP functional, as shown in previous studies.[24, 43, 46]

Although, our quantum-mechanical calculations provide access to all of the predicted vibrational modes involved in the absorption and emission processes of the TMS-capped molecular structures, we have determined that at least for TMS-T4-TMS and TMS-T5-TMS, there are also two vibrations that are the most relevant; the first one around $500 \mathrm{~cm}^{-1}$ and the second one in the region of $1450 \mathrm{~cm}^{-1}$ to $1600 \mathrm{~cm}^{-1}$. However, the situation is different for TMS-T6-TMS, TMST7-TMS, and TMS-T8-TMS because, in these molecules, a low-frequency vibration at around $100 \mathrm{~cm}^{-1}$ also becomes important to the absorption and emission processes. This particular vibration, which shows a relatively large Huang-Rhys factor (see Table S3) introduces an effective spectral broadening of the absorption/emission features of these three compounds, which is in good agreement with previous reports (see for example, Reference [44]). In order to account for the broadening effect of this low-frequency vibration, we have also simulated the absorption/emission spectroscopic profiles of the TMS-T $n$-TMS oligomers by introducing a frequency cut-off that only considers strongly coupled normal modes with frequencies larger than $400 \mathrm{~cm}^{-1}$. The simulated spectral profiles are reported in Figure S3. A simple comparison between the theoretically simulated spectra that used both low- and high-frequency normal modes versus those that only incorporated normal modes with frequencies larger than $400 \mathrm{~cm}^{-1}$ reveals that for the shorter TMS-Tn-TMS ( $n=4$ and 5) oligomers, their spectroscopic 
characteristics are mainly governed by the high-frequency normal modes (no significant differences between the two simulations, see Figure S3). However the simulation comparison suggest that, the larger TMS-Tn-TMS (n=6-8) oligomers require the inclusion of the lowfrequency normal modes modes to account for the moderately broadening and intensity-related observed differences (see Figure S3).

It is also important to note that the $S_{0} \rightarrow S_{1}$ and $S_{1} \rightarrow S_{0}$ Huang-Rhys factors of $\sim 100 \mathrm{~cm}^{-1}$ normal mode become larger as the number of thiophene rings increases. For example, the Huang-Rhys factors related to the absorption transition of this low-frequency vibration along the series of TMS-Tn-TMS oligothienoacenes are: $0.067\left(142 \mathrm{~cm}^{-1}\right), 0.122\left(138 \mathrm{~cm}^{-1}\right), 0.251(128$ $\left.\mathrm{cm}^{-1}\right), 0.345\left(122 \mathrm{~cm}^{-1}\right)$, and $0.411\left(114 \mathrm{~cm}^{-1}\right)$, ongoing fromTMS-T4-TMS to TMS-T8-TMS, respectively. This low-frequency mode stretches the thiophene molecular backbone as a whole along the long molecular axis (see Figure 5). However, these longitudinal stretches are only more pronounced in the larger end-capped oligothienoacenes ( $n=6$ to 8 ), in which the many more internal degrees of freedom, especially those of the outer-most thiophene rings, still distort their respective core-extended transition electron densities, as shown in Figure 5 and Figure S2. 

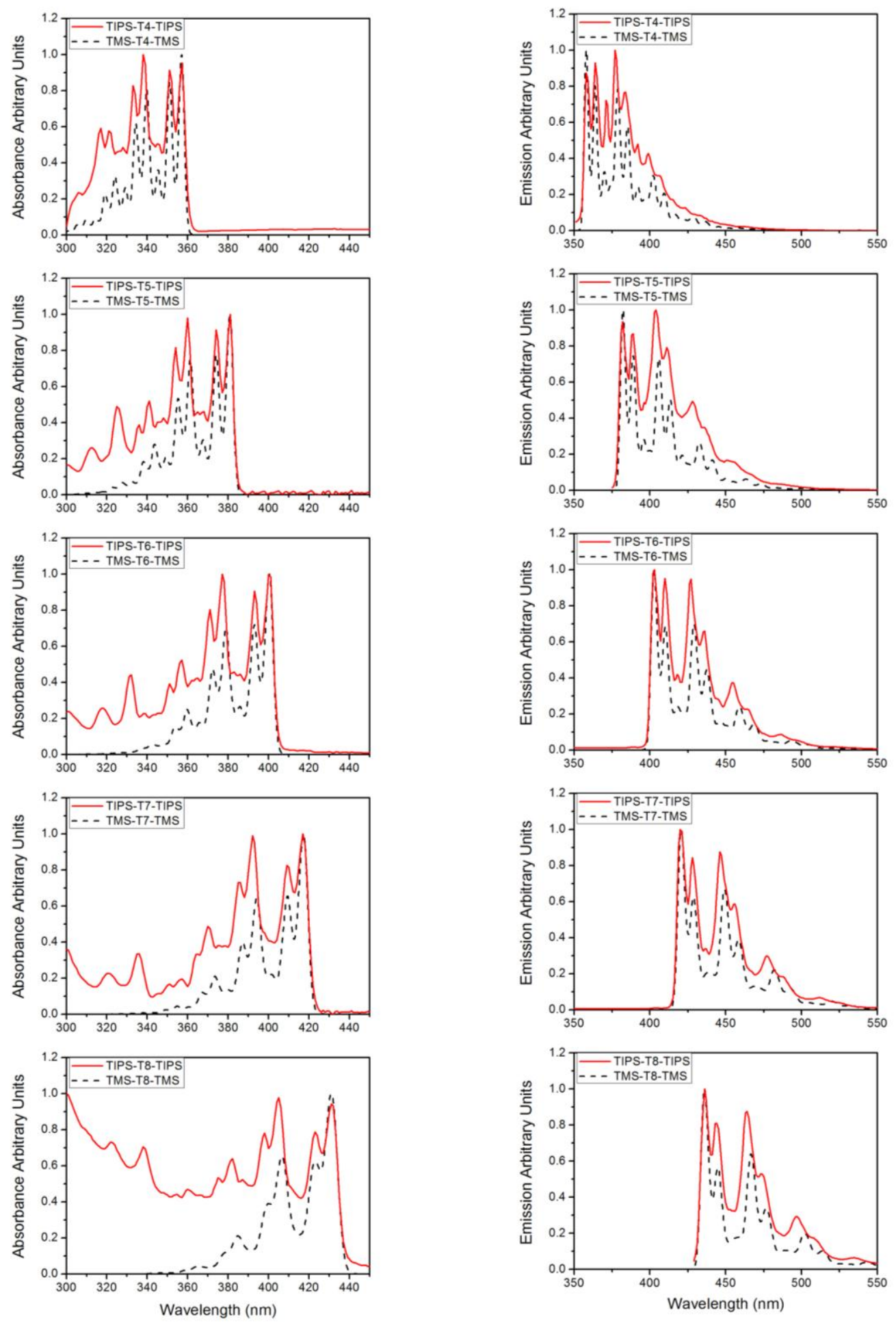

Figure 7. Normalized UV-Vis absorption and fluorescence experimental-spectra of TIPS-TnTIPS (solid lines). The dashed lines represent the simulated (theoretical) spectra convoluted with Gaussian functions. Note that for computational efficiency, in all theoretical simulations we used TMS substituents instead of TIPS substituents. The onset of the simulated spectra is chosen to correspond to experimental onset. 


\section{Conclusions}

This work presents a detailed joint experimental and theoretical analysis of the optical and electronic properties of a family of silyl end-capped oligothienoacenes, which have emerged as a novel class of solution-processable $\pi$-conjugated compounds for organic electronic applications. The experimental absorption and emission profiles showed well-resolved vibronic progressions at both low and room temperature, which is indicative of a minimal conformational disorder of the TIPS-Tn-TIPS oligomers upon electronic transitions. Also, the systematic red-shift of the absorption maxima of the silyl end-capped oligothienoacenes with the extension of the $\pi$ conjugation is in good agreement with the systematic reduction of the calculated HOMO-LUMO gap along the series. When compare to the unsubstituted oligothienoacenes, the insertion of the silyl groups is found to destabilized/stabilized the HOMO/LUMO energies as a consequence of the silicon-induced hyperconjugation effect.

First-principles quantum-mechanical calculations have been used to characterize the experimental absorption and emission profiles of these novel molecular structures. For the smaller molecules (tetramer and pentamer), we found that the vibronic progression of their optical spectra are mainly determined by the presence of two normal modes with frequencies of $\sim 1500 \mathrm{~cm}^{-1}$ and $500 \mathrm{~cm}^{-1}$. However, the larger molecules (hexamer, heptamer, and octamer) are not only characterized by those frequencies but also by the presence of a low-frequency normal mode at around $100 \mathrm{~cm}^{-1}$. We note that this particular vibration is responsible for the experimentally observed peak-broadening of the vibrational absorption and emission features of 
these compounds. Also, our theoretical estimates of the intramolecular reorganization energies upon electronic transition and its subsequent partition into the contributions of each normal mode reveal that the vibronic-coupling interactions in silyl end-capped oligothienoacenes are only associated with their respective oligothienyl molecular backbone.

Finally, our results point that a possible route to explore new soluble organic semiconducting materials should, in principle, include functionalization/modification of their respective molecular backbones. We hope that our results will trigger further experimental investigations for the design of new solution processable organic semiconducting materials. 
Acknowledgements. We thank D. Rappoport and J. Yuen-Zhou for stimulating discussions. We are very grateful to Prof. Adam J. Matzger and Prof. Shigehiro Yamaguchi for kindly providing us the samples. R.S.S-C. thanks the Mary-Fieser Postdoctoral Fellowship at Harvard University. Financial support by the Ministerio de Ciencia e Innovación (MICINN) of Spain (project CTQ2009-10098) and the Junta de Andalucia (grant FQM-0159 and project P06-FQM-1678) are greatly acknowledged. R.M.O. and C.C.F. are also grateful to the UMA and MICINN, respectively, for personal doctoral grants. We are grateful to Dr. Massimo Malagoli (Georgia Institute of Technology, USA) for making available a copy of the program used to simulate the optical spectra. This work was also supported through a National Science Foundation grant via Harvard MRSEC, Grant No. DMR-08-20484. We thank the High Performance Technical Computing Center at the Faculty of Arts and Sciences of Harvard University for invaluable support.

Supporting Information for this article is available on the WWW under http://dx.doi.org/10.1002/chem.2010xxxxx. It contains the normalized (low-temperature) UVVis absorption and fluorescence emission spectra of TMS-T5-TMS and pentathienoacene in MeTHF. Supporting information also includes a Table with selected bond lengths in the groundstate and excited state of TMS-Tn-TMS molecules with $n=5-7$ as determined by DFT/TDDFT/B3LYP calculations; DFT/TD-DFT/B3LYP estimates of frequencies, Huang-Rhys factors, and relaxation energies related to $S_{0} \rightarrow S_{1}$ and $S_{1} \rightarrow S_{0}$ transitions of TMS-Tn-TMS $(n=4,6,7$, and 8); and an illustration of the transition electron densities of TMS-Tn-TMS $(n=4,6$, and 7). Finally, we have also included a Figure that compares the effect of low- and high-frequency 
normal modes versus only high-frequency normal modes in the simulated absorption and fluorescence spectral profiles of the TMS-Tn-TMS $(n=4-8)$ oligomers. 


\section{References}

[1] J.E. Anthony, Angew. Chem., Int. Ed. 47 (2008) 452-483.

[2] S. Henning, Adv. Mater. (Weinheim, Ger.) 21 (2009) 3859-3873.

[3] A.R. Murphy, J.M.J. Frechet, Chem. Rev. 107 (2007) 1066-1096.

[4] J. Roncali, Acc. Chem. Res. 42 (2009) 1719-1730.

[5] T. Okamoto, K. Kudoh, A. Wakamiya, S. Yamaguchi, Chem. Eur. J. 13 (2007) 548-556.

[6] X.N. Zhang, A.P. Cote, A.J. Matzger, J. Am. Chem. Soc. 127 (2005) 10502-10503.

[7] E.-G. Kim, V. Coropceanu, N.E. Gruhn, R.S. Sánchez-Carrera, R. Snoeberger, A.J. Matzger, J.-L. Brédas, J. Am. Chem. Soc. 129 (2007) 13072-13081.

[8] X.N. Zhang, J.P. Johnson, J.W. Kampf, A.J. Matzger, Chem. Mater. 18 (2006) 3470-3476.

[9] O.L. Griffith, N.E. Gruhn, J.E. Anthony, B. Purushothaman, D.L. Lichtenberger, J. Phys. Chem. C 112 (2008) 20518-20524.

[10] R.M. Osuna, M.C.R. Delgado, V. Hernandez, J.T.L. Navarrete, B. Vercelli, G. Zotti, J.J. Novoa, Y. Suzuki, S. Yamaguchi, J.T. Henssler, A.J. Matzger, Chem. Eur. J. 15 (2009) 12346-12361.

[11] R.M. Osuna, V. Hernandez, J.T.L. Navarrete, J. Arago, P.M. Viruela, E. Orti, Y. Suzuki, S. Yamaguchi, J.T. Henssler, A.J. Matzger, ChemPhysChem 10 (2009) 3069-3076.

[12] M. Klessinger, J. Michl, Excited states and photochemistry of organic molecules, VCH, New York, 1995.

[13] J.R. Lakowicz, Principles of fluorescence spectroscopy, 2nd ed., Kluwer Academic/Plenum, New York, 1999.

[14] V. Navarro-Fuster, E.M. Calzado, M.G. Ramirez, P.G. Boj, J.T. Henssler, A.J. Matzger, V. Hernandez, J.T.L. Navarrete, M.A. Diaz-Garcia, J. Mater. Chem. 19 (2009) 6556-6567.

[15] J. Aragó, P.M. Viruela, E. Ortí, R.M. Osuna, B. Vercelli, G. Zotti, V. Hernández, J.T. López Navarrate, J.T. Henssler, A.J. Matzger, Y. Suzuki, S. Yamaguchi, Chem. Eur. J. 16 (2010) 5481-5491.

[16] J.L. Brédas, D. Beljonne, V. Coropceanu, J. Cornil, Chem. Rev. 104 (2004) 4971-5003.

[17] M. Malagoli, V. Coropceanu, D.A. da Silva Filho, J.L. Brédas, Journal of Chemical Physics 120 (2004) 7490-7496.

[18] P.F. Barbara, T.J. Meyer, M.A. Ratner, J. Phys. Chem. 100 (1996) 13148-13168.

[19] J.R. Reimers, Journal of Chemical Physics 115 (2001) 9103-9109.

[20] M.W. Wong, Chem. Phys. Lett. 256 (1996) 391-399.

[21] Z. Zhao, F.C. Spano, J. Phys. Chem. C 111 (2007) 6113-6123.

[22] F. Furche, R. Ahlrichs, J. Chem. Phys. 117 (2002) 7433-7447.

[23] D. Rapport, F. Furche, Time-Dependent Density Functional Theory, Springer, Berlin, 2006, pp. 337-357.

[24] M. Dierksen, S. Grimme, J. Phys. Chem. A 108 (2004) 10225-10237.

[25] M. Dierksen, S. Grimme, J. Chem. Phys. 120 (2004) 3544-3554.

[26] A.D. Becke, J. Chem. Phys. 98 (1993) 5648-5652.

[27] C.T. Lee, W.T. Yang, R.G. Parr, Phys. Rev. B 37 (1988) 785-789.

[28] M.M. Francl, W.J. Pietro, W.J. Hehre, J.S. Binkley, M.S. Gordon, D.J. Defrees, J.A. Pople, J. Chem. Phys. 77 (1982) 3654-3665.

[29] P.C. Harihara, J.A. Pople, Theor. Chim. Acta 28 (1973) 213-222.

[30] W.J. Hehre, Ditchfie.R, J.A. Pople, J. Chem. Phys. 56 (1972) 2257-2261. 
[31] TURBOMOLE V6.1 2009 a development of University of Karlsruhe and Forschungszentrum Karlsruhe GmbH, 1989-2007, TURBOMOLE GmbH, since 2007; available from http://www.turbomole.com.

[32] Y. Suzuki, T. Okamoto, A. Wakamiya, S. Yamaguchi, Org. Lett. 10 (2008) 3393-3396.

[33] G. Macchi, B. Milian Medina, M. Zambianchi, R. Tubino, J. Cornil, G. Barbarella, J. Gierschner, F. Meinardi, Phys. Chem. Chem. Phys. 11 (2009) 984-990.

[34] E. Leontidis, U.W. Suter, M. Schuetz, H.-P. Luethi, A. Renn, U.P. Wild, J. Am. Chem. Soc. 117 (1995) 7493-7507.

[35] A.M. Stoneham, Rev. Mod. Phys. 41 (1969) 82-108.

[36] D. Wasserberg, S.C.J. Meskers, R.A.J. Janssen, E. Mena-Osteritz, P. Bauerle, J. Am. Chem. Soc. 128 (2006) 17007-17017.

[37] D.M. Wetzel, J.I. Brauman, J. Am. Chem. Soc. 110 (1988) 8333-8336.

[38] C. Risko, G.P. Kushto, Z.H. Kafati, J.L. Brédas, J. Chem. Phys. 121 (2004) 9031-9038.

[39] X. Zhan, C. Risko, F. Amy, C. Chan, W. Zhao, S. Barlow, A. Kahn, J.L. Brédas, S.R. Marder, J. Am. Chem. Soc. 127 (2005) 9021-9029.

[40] G. Lu, H. Usta, C. Risko, L. Wang, A. Facchetti, M.A. Ratner, T.J. Marks, J. Am. Chem. Soc. 130 (2008) 7670-7685.

[41] S. Yamaguchi, Y. Itami, K. Tamao, Organometallics 17 (1998) 4910-4916.

[42] G.R. Hutchison, M.A. Ratner, T.J. Marks, J. Am. Chem. Soc. 127 (2005) 2339-2350.

[43] R.S. Sánchez-Carrera, V. Coropceanu, D.A. da Silva Filho, R. Friedlein, W. Osikowicz, R. Murdey, C. Suess, W.R. Salaneck, J.L. Brédas, J. Phys. Chem. B 110 (2006) 18904-18911.

[44] S. Karabunarliev, M. Baumgarten, E.R. Bittner, K. Mullen, J. Chem. Phys. 113 (2000) 1137211381.

[45] G. Orlandi, F. Zerbetto, M.Z. Zgierski, Chem. Rev. 91 (1991) 867-891.

[46] K. Schmidt, S. Brovelli, V. Coropceanu, J.L. Brédas, C. Bazzini, T. Caronna, R. Tubino, F. Meinardi, J. Phys. Chem. A 110 (2006) 11018-11024. 